International Journal of Wireless \& Mobile Networks (IJWMN) Vol. 5, No. 1, February 2013

\title{
Speech Enhancement Algorithm to Reduce the Effect of Background Noise in Mobile Phones
}

\author{
Premananda B S ${ }^{1}$ and Dr. Uma B V ${ }^{2}$ \\ ${ }^{1}$ Department of Telecommunication Engineering, \\ R.V. College of Engineering, Bangalore, India. \\ premanandabservce.edu. in \\ ${ }^{2}$ Department of Electronics and Communication Engineering, \\ R.V. College of Engineering, Bangalore, India.
}

umabvarvce.edu.in

\begin{abstract}
In mobile phones, perceived quality of speech signal deteriorates significantly in the presence of background noise since near-end/surrounding noise also arrives at the near-end listener's ears. The quality of the received signal varies widely depending upon signal strength and unavoidable background noise in the user environment. There is a need to improve the quality of received speech signal in noisy conditions by developing the speech enhancement algorithms. This paper focuses on the impact of the various background noises on signal degradation and mechanisms to mitigate the noise impact for improved speech signal perception. Gain adjustment process with simple time domain and frequency domain approach using psychoacoustic has been adapted to improve the quality and intelligibility of the speech signal in the noisy environments by automatically enhancing the speech signals when the noise dominates. Time domain approach is less complex and frequency domain using psychoacoustic is more efficient in overcoming the degradation of speech signals in presence of near-end or background noise.
\end{abstract}

\section{KEYWORDS}

Absolute Threshold of Hearing, Background noise, Degradation, Gain, Psychoacoustic, Speech enhancement.

\section{INTRODUCTION}

Mobile phones are essential component of day to day life and have become the best friend of a person. Day by day they are becoming very affordable making the communication infrastructure as the backbone of daily life. Background noise is a natural part of a conversation. A high background acoustic noise level is annoying to the listener side. Listener fatigue (the ears get tired) and difficult to understand each other. Considerable attention has been paid over the past decade for the enhancement of speech degraded by additive background noise [6]. Listening to speech or audio signals becomes more difficult as the background noise level dominates. The near-end listener perceives a combination of the clean far-end (downlink) speech and the background noise from the near-end and thus experiences an increased listening effort. As the noise signal cannot be influenced, a reasonable approach is to manipulate the clean far-end speech signal depending on the local background noise. Hence, there is a strong need to improve the quality of the speech signal in noisy conditions by developing speech enhancement algorithms to minimize the effect of background noise. In the gain adjustment process the quality of the speech signal in noisy environment is improved by automatically adjusting the output level when the background noise exceeds the noise masking threshold. 
International Journal of Wireless \& Mobile Networks (IJWMN) Vol. 5, No. 1, February 2013

The traditional noise cancellation algorithms cannot be used as the near-end noise directly arrives at the near-end listener's ears. The approaches proposed for far-end noise cancellation discussed in literature [1], [2], [3], [4] are not suitable. In the subtractive-type approach proposed by Virag [1], a psychoacoustic model was used to guide the derivation of the spectral subtractive parameters. Jeon Yu-young et al. discusses about the compensation for partial masking effect [5]. Several approaches to mitigate the background (near-end) noise using speech enhancement are discussed by Bastian et al. [6], [7], [8], [9] and Jong Won Shin et al. [10], [11], [12]. Speech intelligibility improvement in presence of near-end noise and loudspeaker output power constraint are discussed in [6], [7]. Perceptual speech reinforcement based on partial specific loudness is discussed in [10], [11] focuses on mitigating near end noise at the speaker not at the receiver. Psychoacoustic model in frequency domain approach using waveletthreshold multitaper magnitude spectra as alternate to Fast Fourier Transform (FFT) was incorporated by $\mathrm{Yi} \mathrm{Hu} \mathrm{[13].} \mathrm{The} \mathrm{speech} \mathrm{enhancement} \mathrm{methods} \mathrm{today} \mathrm{are} \mathrm{more} \mathrm{exploiting} \mathrm{the}$ auditory masking properties of human [3]. Many approaches discussed above have not fully exploited the psychoacoustic approach for speech enhancement in presence of near-end noise.

The paper is organized as follows: In section 2, proposed time domain approach is described with implementation details and results. Section 3, describes the proposed frequency domain approach using psychoacoustic with the implementation details and results. Comparison results of both the approaches are discussed in Section 4, and the conclusions are given in Section 5.

\section{Proposed Time Domain Speech Enhancement Method}

This paper proposes a method to overcome the relative degradation of the speech signal in the noisy environment. In order to retain the signal quality in the noisy environment different algorithms have been proposed and implemented in [6-9], but the complexity involved in determining the gain seems to be unresolved. In this paper a concept which is simple to analyze and implement in real time is presented. A multiplier is required to enhance the speech signals degraded in the presence of background noise. As the speech and noise signals are dynamic it is difficult to find a constant multiplication factor (MF) for the incoming signal. Thus the signal strength and noise present in the environment is to be analyzed. The speech signal is to be amplified by applying a dynamically varying gain depending upon the different parameters of speech and noise signal. The external volume control of the devices can't be used for this purpose as it is painful.

Block diagram of the proposed time domain approach is shown in the figure 1. Energy of the downlink speech signal and near-end noise signal is computed. By comparing energy of both the speech and noise signals, gain/MF is computed for enhancing the speech signal. Gain obtained is multiplied with speech (element by element) signal to get enhanced speech signal.

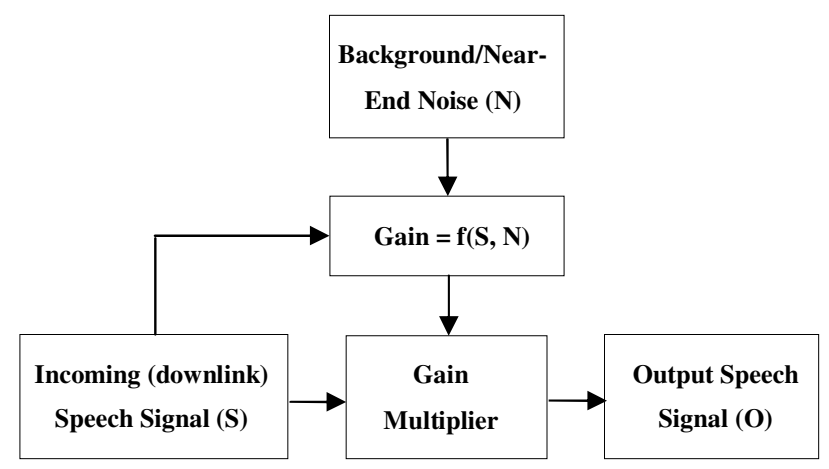

Fig. 1: Proposed time domain approach. 
The sound energy is proportional to the square of the amplitude. Energy, $\mathrm{E}_{\mathrm{dB}}$ in decibels is calculated using,

$$
\mathrm{E}_{\mathrm{dB}}=10 * \log _{10} \sum\left(\mathrm{X}^{2}\right) / \mathrm{N}
$$

where $\mathrm{X}$ is the amplitude of the speech signal and $\mathrm{N}$, number of samples.

The Gain is to be adaptive for being effective in most of the scenarios. It can be modelled as,

$$
\text { Gain }=\sum_{i=1}^{p} c_{i} x_{i}
$$

where $\mathrm{x}_{1} \ldots \mathrm{x}_{\mathrm{i}} \ldots \mathrm{x}_{\mathrm{p}}$ represent different parameters and $\mathrm{c}_{1} \ldots \mathrm{c}_{\mathrm{i}} \ldots \mathrm{c}_{\mathrm{p}}$ represent different coefficients to be derived corresponding to one or more of the above mentioned parameters.

The challenge lies in finding out the correct coefficients for adapting equation (2). Hit and trial methods or human expertise can be utilized for setting these values, works for limited categories of speech signals and noises.

\subsection{Deriving the Gain}

The correct gain/MF for a pair of speech and noise sample (selected parameters) is user specific. System provides a mechanism for user to declare whether system generated MF (auto gain) is acceptable or not. Such an assumption is easy to realize for most of the devices as volume control can be used for the purpose. In figure 1, gain block calculates the amplification factor/MF of speech signal in the presence of varying background noise which can dominate the received speech signal as well. Different parameters of signal and noise (energy in the present case) can be considered. In order to avoid the perceptual artefacts like clicks \& pops due to signal saturation/ overflows and signal bursts due to sudden gain changes, optimal gain computed must be characterized by slow and configurable response time for the gain variations.

The Gain is derived using the equation,

$$
\text { Gain }=\mathrm{A}+\operatorname{maximum}(\mathrm{B},(\mathrm{C}-\mathrm{D})) \cdot \mathrm{E}
$$

where A, B, C, D, and E are experimental constants. Value of A (default gain) is set to 1 so that Gain $=1$ when no enhancement for the speech signal is required, B (default enhancement) is set to 0 . (C - D) is extent of noise over speech signal, (if negative no amplification is required), $\mathrm{E}$ (compensation factor) is used to control the gain $(<1)$.

When signal energy (SE) is sufficiently greater than noise energy (NE) then gain of 1 is used so that no amplification is required, input signal buffer value will be copied to output signal buffer. When SE is approximately equal to NE then gain is selected such that enhanced speech signal is increased nearly by $1 \mathrm{~dB}$. When SE is less than NE then gain is calculated using eq. 3. Present frame gain/MF is compared with previous frame MF if the difference is more, then current frame MF is adjusted to avoid sudden change in large gain to avoid click and pop noise. If the hearing and perception choice of user varies with time, the proposed mechanism is capable of capturing such alterations. If the amplification value exceeds the maximum loudness [7] of the loud speaker [9] then end capping can be performed depending on minimum and maximum values computed using,

Case-1: for +ve amplified value (AV)

$\mathrm{AV}=\operatorname{minimum}\left(\mathrm{AV}, 2^{15}-1\right)$

Case-2: for -ve amplified value (AV)

$$
\mathrm{AV}=\operatorname{maximum}\left(\mathrm{AV},-2^{15}\right)
$$


International Journal of Wireless \& Mobile Networks (IJWMN) Vol. 5, No. 1, February 2013

Signal buffer value can be overridden with new amplified value and output to output speech signal buffer. Most mobile phone applications limit the overall power of the loudspeaker signal to a constant maximum power instead of the power of the original signal.

\subsection{Experimental Results}

Algorithm has been verified for different speech signals with varying background (tapering, hammer, parabolic type ...) noise signals. To illustrate a speech signal of 16-bit PCM with sampling rate of $8 \mathrm{kHz}$ with duration of 5 seconds is considered. The speech signal is captured using an audio editor tool Gold Wave and saved in .wav format. The captured speech signal has $40000(8000 * 5)$ samples, total samples are divided into frame size of 1024 each, resulting in 39 frames. Varying background noise (Fig. 2(b)) for 5 seconds, sampled at $8 \mathrm{kHz}$ is captured using an audio editor tool Gold Wave and saved in .wav format for experimenting and gain/MF are calculated. Microphone in the mobile phones can be used to capture the background noise signals. Experiment is performed by considering the difference in energy of background noise $(\mathrm{dB})$ and speech signal $(\mathrm{dB})$. Figure 2 show the results obtained for varying background noise. The speech signal in figure 2(a) corresponds to input (downlink) speech signal, figure 2(b) represents noise signal whose amplitude is continuously decreasing, and figure 2(c) shows the amplified speech signal in presence of noise where the gain is varied from 1 to 7 .

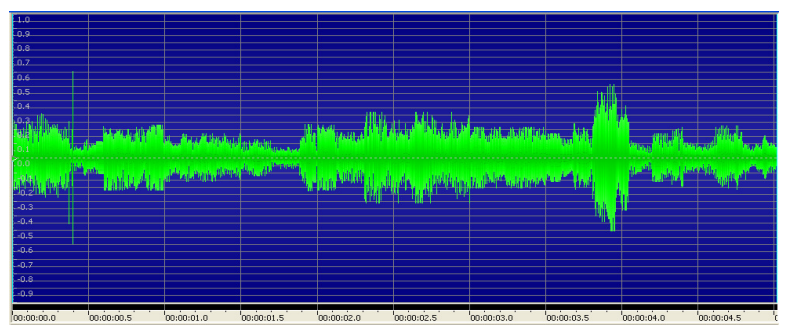

Fig. 2(a): Original down linked speech signal.

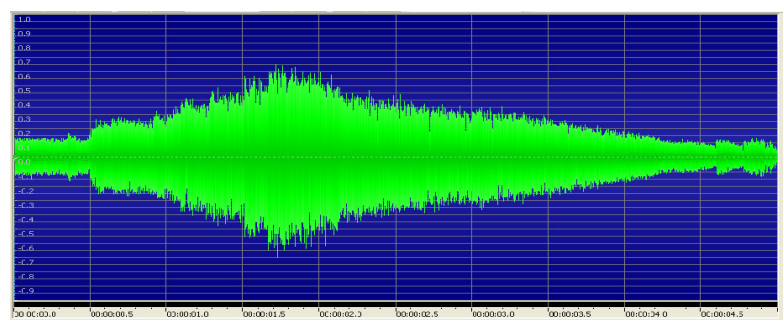

Fig. 2(b): Varying background noise signal.

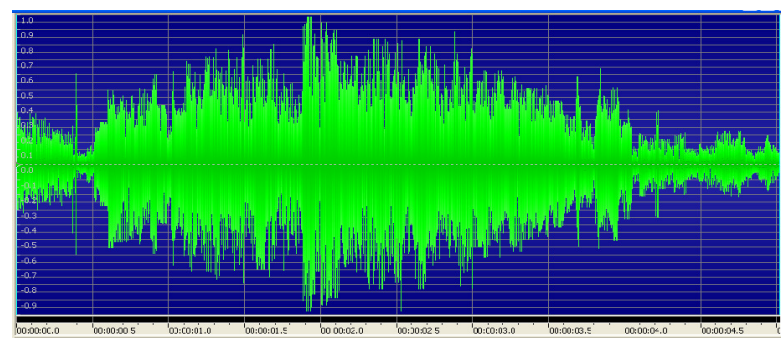

Fig. 2(c): Enhanced speech signal using time domain approach. 
International Journal of Wireless \& Mobile Networks (IJWMN) Vol. 5, No. 1, February 2013

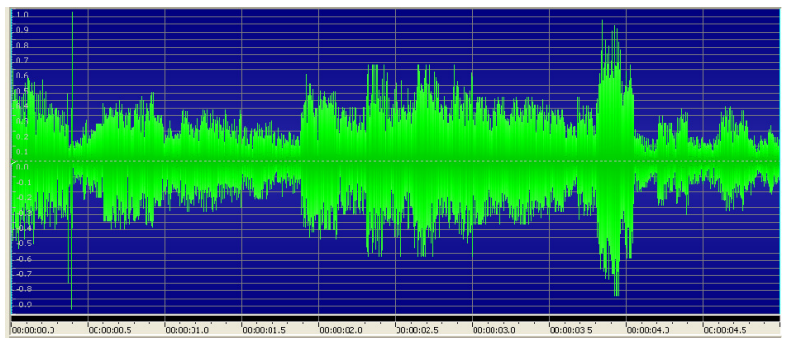

Fig. 2(d): Enhanced speech signal using frequency domain approach.

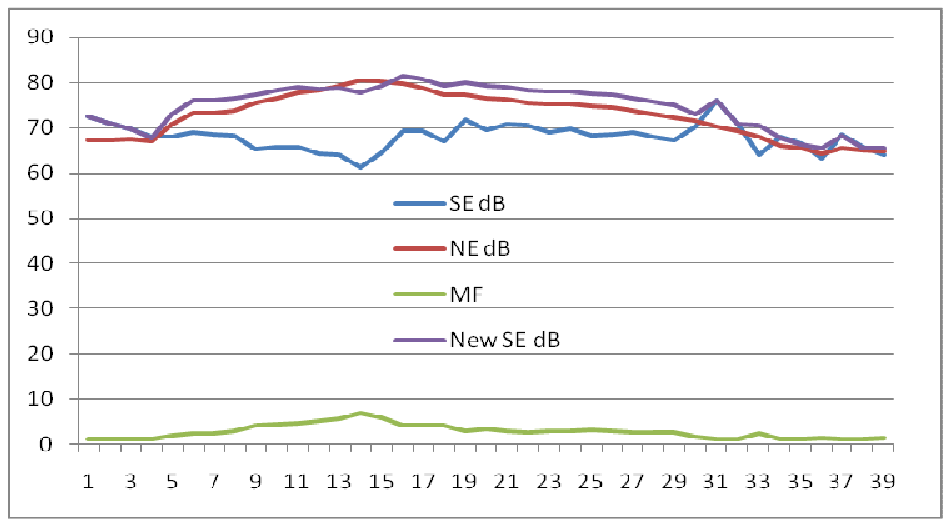

Fig. 3: Variations of speech, noise, enhanced speech signal and gain/MF using time domain approach.

Figure 2(c) reflects the variation in the amplification of speech signal based on changing noise signals. Speech signal can be further enhanced by increasing the value of gain control, E in equation 3 as desired. The obtained results are plotted in figure 3 to verify how dynamically gain/MF is varied in accordance with speech and noise signals. Number of frames is indicated in $\mathrm{x}$-axis and $\mathrm{y}$-axis indicates energy of the speech, noise \& enhanced signal and variation of gain w.r.t. speech and noise signal. For a gain of $\approx 7$, when the noise signal is at maximum, the speech signal energy is enhanced by $\approx 17 \mathrm{~dB}$. Speech signal has enhanced to a maximum energy of $\approx 82 \mathrm{~dB}$.

\section{Proposed Frequency Domain Speech Enhancement Method}

\subsection{Psychoacoustics}

Psychoacoustics is the study of sound perception (including speech and music) [1], [7]. The range of human hearing is about $20 \mathrm{~Hz}$ to $20 \mathrm{kHz}$ and frequency range of the speech (voice) is typically from $500 \mathrm{~Hz}$ to $4 \mathrm{kHz}$. The dynamic range of sound (ratio of the maximum sound amplitude to the quietest sound that can be heard), is of the order of about $120 \mathrm{~dB}$ [14]. Equalloudness contours indicate the sound pressure level $(\mathrm{dB})$, over the range of audible frequencies, which are perceived as being of equal loudness. Equal-loudness contours were first measured by Fletcher and Munson using pure tones reproduced via headphones, and the data collected are called Fletcher-Munson curves.

Speech enhancement methods today are more often exploiting the human auditory masking properties [3]. Yi $\mathrm{Hu}$ et al. proposes speech enhancement in the frequency domain as a constrained minimization problem and includes the masking thresholds as the constraints. In [13], psychoacoustic model is integrated in the derived spectral weighting function, paper further investigate the importance of using good (low variance) spectrum estimators in speech 
International Journal of Wireless \& Mobile Networks (IJWMN) Vol. 5, No. 1, February 2013

enhancement. The human listener will not perceive any noise distortion as long as the power spectrum density of the distortion lies below the masking threshold (the masking thresholds can be obtained by performing critical band analysis of the speech signal [13]). As an alternative to time domain approach (section 2) is to amplify the frequency components of the signal so that the noise level in each critical band becomes lower than the masking threshold. In most of the above speech enhancement methods, the incorporation of auditory masking was done heuristically.

\section{A. Absolute Threshold of Hearing}

ATH is minimum sound level of a pure tone that an average ear with normal hearing can hear with no other sound present, this is also known as the auditory threshold. It is the lowest of the equal-loudness contours. If one has a low absolute threshold, it means that he is able to detect small amounts of stimulation, and thus is more sensitive. If one has a high absolute threshold, then he requires more stimulation and thus is less sensitive [14]. ATH varies with the frequency of the sound, which is shown in figure 4.

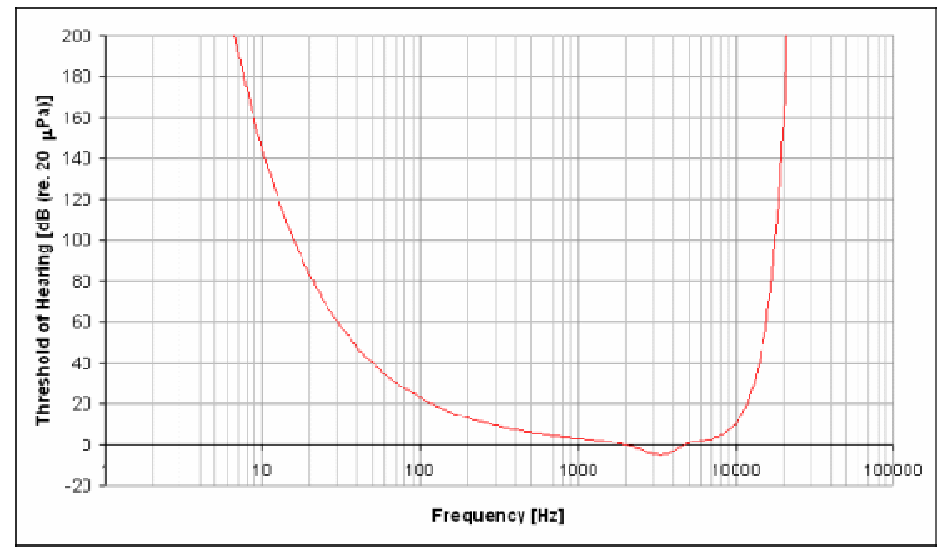

Fig. 4: Threshold of hearing.

The threshold of hearing is given by,

$$
T_{q}(f)=3.64\left(\frac{f}{1000}\right)^{-0.8}-6.5 e^{-0.6\left(\frac{f}{1000}-3.3\right)^{2}}+10^{-3}\left(\frac{f}{1000}\right)^{4}
$$

where

$$
T_{q}(f)=\text { Threshold of Hearing }(\mathrm{dB}) \text { and } f=\text { frequency }(\mathrm{Hz})
$$

The threshold of hearing is generally reported as the RMS sound pressure of $20 \mu \mathrm{Pa}$. It is the sound a young human with undamaged hearing can detect at $1000 \mathrm{~Hz}$. The threshold of hearing is frequency dependent and it has been shown that the ear's sensitivity is best at frequencies between $1 \mathrm{kHz}$ to $5 \mathrm{kHz}$ [14].

\section{B. Masking}

The masking phenomenon occurs because any loud sound will distort the ATH, making quieter, otherwise perceptible sounds inaudible [14]. The masking effect stands for the phenomenon that a certain weak signal called a maskee cannot be heard, i.e., 'masked' in the presence of a strong signal called a masker in a nearby time or frequency region [12]. A weaker sound is masked if it is made inaudible in the presence of a louder sound. The stronger signal that masks the weaker one is called masker and the one that is masked is the maskee. The masking threshold will depend upon the sound pressure level (SPL), the frequency of the masker and the characteristics of the masker and the maskee, such as whether the masker or maskee is a tone or noise. The masking characteristics are measured by signal to mask ratio (SMR) and mask to noise ratio 
International Journal of Wireless \& Mobile Networks (IJWMN) Vol. 5, No. 1, February 2013

(MNR). SMR at a given frequency is expressed as the difference (in $\mathrm{dB}$ ) between the sound pressure level of the masker and the masking threshold at that frequency. MNR at a given frequency is expressed as the difference (in $\mathrm{dB}$ ) between the masking threshold at that frequency and the noise level. To make the noise inaudible, its level should be below the masking threshold i.e. the MNR should be positive [3].

If there is more than one masker, then each masker contributes to its own masking threshold and global masking threshold is computed that describes just noticeable distortion as a function of frequency.

Simultaneous Masking: masking between two concurrent sounds often observed when the sounds share a frequency band.

Temporal Masking: characteristic of the auditory system where sounds are hidden due to maskers which have just disappeared, or even after maskers which are about to appear.

Loudness: Sound loudness is a subjective term describing the strength of the ear's perception of a sound, also referred to as volume or strength. The units used to measure loudness are the, Sone (loudness N) and Phon (loudness level L).

\subsection{Deriving the Weighted Curve using Psychoacoustic}

In the measurement of loudness, weighted curve is used to emphasize frequencies around 3 to 5 $\mathrm{kHz}$ where the human ear is most sensitive, while attenuating very low and high frequencies to which the ear is insensitive [14]. Deriving a weighted curve is considered as one of the most important factors which facilitates in measuring noise loudness. Table I shows the derived threshold of hearing (dBSPL) values using equation 6, for frequencies ranging from $20 \mathrm{~Hz}$ to 20 $\mathrm{kHz}$ (frequency range for human hearing). In table I, the column I represent 64 different frequency values ranging from $20 \mathrm{~Hz}$ to $20 \mathrm{KHz}$, and column 2 represents threshold of hearing computed $(\mathrm{dB})$ for the respective frequencies.

The linear curve (figure 5) is derived for 64 vectors, with a fixed linear frequency interval of $312.5 \mathrm{~Hz}$ along the $\mathrm{X}$ axis, using the equation 6 . The threshold of the hearing was found to be $-4.983187589 \mathrm{~dB}$ at $3320.3125 \mathrm{~Hz}$, and $160.3313427 \mathrm{~dB}$ at $20 \mathrm{kHz}$ which shows, our ear is most sensitive to the frequency around 3.3 to $3.5 \mathrm{kHz}$. It is observed that maximum audibility lies in the range of 1 to $5 \mathrm{kHz}$; any signal with least loudness can be heard in this range.

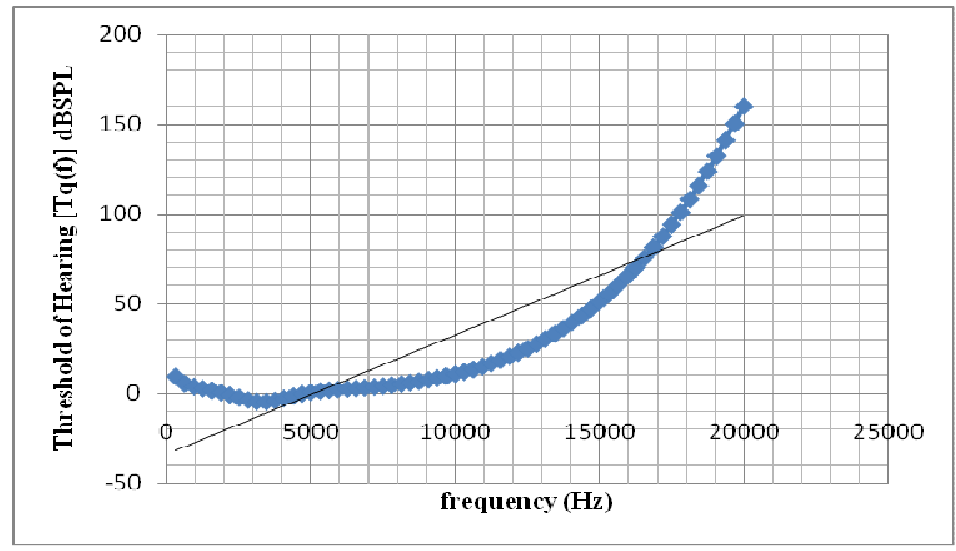

Fig. 5: Threshold of Human Hearing.

After deriving 64 Vectors for a frequency range of $20 \mathrm{~Hz}$ to $20 \mathrm{kHz}$, its weighted curve has to be derived, for this we have normalized 64 vectors to lie in between 0 and 1 (figure 6). To normalize these values we use equation 7 to set maximum value $160.3313427 \mathrm{~dB}$ (threshold) of $20 \mathrm{kHz}$ to be 1 and minimum value $-4.931530782 \mathrm{~dB}$ of $3.4375 \mathrm{KHz}$ to 0 . 
International Journal of Wireless \& Mobile Networks (IJWMN) Vol. 5, No. 1, February 2013

$$
X^{\prime}(\mathrm{n})=\frac{X(n)-\min (\text { score })}{\max (\text { score })}
$$

where,

$\mathrm{X}^{\prime}(\mathrm{n})=$ Normalized value, $X(\mathrm{n})=$ Value to be normalized, $\min ($ score $)=$ minimum value, $\max ($ score $)=$ maximum value.

The weighed curve is the inverse of Threshold of hearing curve shown in figure 6, normalized between 0 and 1 . We take $1-X^{\prime}(n)$ (represented as $B(n)$ in table I), to derive the weighed curve shown in figure 7.

Table 3.1: $\mathrm{F}=$ frequency $(\mathrm{Hz})$ [column I], Tq(f) = Threshold of Hearing (dB) [column II], $\mathrm{X}^{\prime}(\mathrm{n})=$ Normalized Values [column III], B(n) = 1-X'(n) [column IV].

\begin{tabular}{|c|c|c|c|c|c|c|c|c|c|}
\hline No. & $\begin{array}{c}\text { I } \\
\text { Freq. (Hz) }\end{array}$ & $\begin{array}{c}\text { II } \\
\mathbf{T q}(\mathbf{f})\end{array}$ & $\begin{array}{c}\text { III } \\
X^{\prime}(n)\end{array}$ & $\begin{array}{c}\text { IV } \\
\text { B(n) }\end{array}$ & No. & $\begin{array}{c}\text { I } \\
\text { Freq. (Hz) }\end{array}$ & $\begin{array}{c}\text { II } \\
\text { Tq(f) }\end{array}$ & $\begin{array}{c}\text { III } \\
\mathbf{X}^{\prime}(\mathbf{n})\end{array}$ & $\begin{array}{c}\text { IV } \\
\text { B(n) }\end{array}$ \\
\hline 1 & 312.5 & 9.199721317 & 0.085507724 & 0.914492276 & 33 & 10312.5 & 11.87269673 & 0.101681806 & 0.898318194 \\
\hline 2 & 625 & 5.212853678 & 0.061383324 & 0.938616676 & 34 & 10625 & 13.29388247 & 0.110281353 & 0.889718647 \\
\hline 3 & 937.5 & 3.605332751 & 0.05165627 & 0.948343731 & 35 & 10937.5 & 14.84806612 & 0.119685665 & 0.880314335 \\
\hline 4 & 1250 & 2.525140233 & 0.045120061 & 0.954879939 & 36 & 11250 & 16.54309066 & 0.1299422 & 0.870057821 \\
\hline 5 & 1562.5 & 1.490733319 & 0.038860901 & 0.961139099 & 37 & 11562.5 & 18.38703346 & 0.141099835 & 0.858900165 \\
\hline 6 & 1875 & 0.291649746 & 0.031605287 & 0.968394713 & 38 & 11875 & 20.38820559 & 0.15320886 & 0.846791141 \\
\hline 7 & 2187.5 & -1.12430887 & 0.02303737 & 0.976962631 & 39 & 12187.5 & 22.55515119 & 0.166320973 & 0.833679027 \\
\hline 8 & 2500 & -2.63945460 & 0.013869275 & 0.986130725 & 40 & 12500 & 24.8966472 & 0.180489284 & 0.819510716 \\
\hline 9 & 2812.5 & -3.98204419 & 0.005745311 & 0.994254689 & 41 & 12812.5 & 27.42170193 & 0.195768306 & 0.804231694 \\
\hline 10 & 3125 & -4.82336305 & 0.000654519 & 0.999345481 & 42 & 13125 & 30.13955663 & 0.212213951 & 0.787786049 \\
\hline 11 & 3437.5 & -4.93153078 & 5.37434E-18 & 1 & 43 & 13437.5 & 33.05968325 & 0.229883538 & 0.770116462 \\
\hline 12 & 3750 & -4.29421157 & 0.003856397 & 0.996143603 & 44 & 13750 & 36.19178507 & 0.248835779 & 0.751164221 \\
\hline 13 & 4062.5 & -3.12744206 & 0.010916479 & 0.989083521 & 45 & 14062.5 & 39.54579634 & 0.269130786 & 0.730869214 \\
\hline 14 & 4375 & -1.76521239 & 0.019159284 & 0.980840716 & 46 & 14375 & 43.13188198 & 0.290830068 & 0.709169932 \\
\hline 15 & 4687.5 & -0.50721883 & 0.02677136 & 0.97322864 & 47 & 14687.5 & 46.96043748 & 0.313996527 & 0.686003473 \\
\hline 16 & 5000 & 0.481694717 & 0.032755243 & 0.967244757 & 48 & 15000 & 51.04208865 & 0.338694458 & 0.661305542 \\
\hline 17 & 5312.5 & 1.181223029 & 0.036988064 & 0.963011936 & 49 & 15312.5 & 55.38769156 & 0.364989553 & 0.635010447 \\
\hline 18 & 5625 & 1.66154186 & 0.039894457 & 0.960105543 & 50 & 15625 & 60.00833233 & 0.392948893 & 0.607051107 \\
\hline 19 & 5937.5 & 2.018207613 & 0.04205263 & 0.95794737 & 51 & 15937.5 & 64.91532708 & 0.42264095 & 0.577359051 \\
\hline 20 & 6250 & 2.331012374 & 0.0439454 & 0.95605461 & 52 & 16250 & 70.12022178 & 0.45413559 & 0.545864411 \\
\hline 21 & 6562.5 & 2.651831043 & 0.045886663 & 0.954113337 & 53 & 16562.5 & 75.63479221 & 0.487504067 & 0.512495933 \\
\hline 22 & 875 & 3.009545465 & 0.048051181 & 0.951948819 & 54 & 16875 & 81.47104384 & 0.522819026 & 0.477180974 \\
\hline 23 & 7187.5 & 3.419366485 & 0.050530994 & 0.949469006 & 55 & 17187.5 & 87.64121176 & 0.560154502 & 0.439845498 \\
\hline 24 & 7500 & 3.89009146 & 0.053379335 & 0.946620665 & 56 & 17500 & 94.15776064 & 0.599585916 & 0.400414084 \\
\hline 25 & 7812.5 & 4.42811903 & 0.056634921 & 0.943365079 & 57 & 17812.5 & 101.0333847 & 0.641190082 & 0.358809918 \\
\hline 26 & 8125 & 5.039207051 & 0.060332594 & 0.939667406 & 58 & 18125 & 108.2810075 & 0.6850452 & 0.31495482 \\
\hline 27 & 8437.5 & 5.729107249 & 0.064507156 & 0.935492844 & 59 & 18437.5 & 115.9137821 & 0.731230858 & 0.268769142 \\
\hline 28 & 8750 & 6.503757032 & 0.069194536 & 0.930805464 & 60 & 18750 & 123.9450909 & 0.779828034 & 0.220171966 \\
\hline 29 & 9062.5 & 7.369327338 & 0.074432072 & 0.925567928 & 61 & 19062.5 & 132.3885457 & 0.83091909 & 0.16908092 \\
\hline 30 & 9375 & 8.33223132 & 0.080258571 & 0.919741429 & 62 & 19375 & 141.2579876 & 0.88458778 & 0.11541222 \\
\hline
\end{tabular}


International Journal of Wireless \& Mobile Networks (IJWMN) Vol. 5, No. 1, February 2013

\begin{tabular}{|l|c|c|c|c|c|c|c|c|c|}
\hline 31 & 9687.5 & 9.39912406 & 0.086714303 & 0.913285697 & 63 & 19687.5 & 150.5674867 & 0.940919241 & 0.059080759 \\
\hline 32 & 10000 & 10.57690112 & 0.093840991 & 0.906159009 & 64 & 20000 & 160.3313427 & 1 & $1.98947 \mathrm{E}-10$ \\
\hline
\end{tabular}

\subsection{Deriving the Gain}

Deriving the gain in frequency domain approach is more critical than time domain approach. Background noise is captured and sampled at a rate of $8 \mathrm{kHz}$. The total samples are divided into frame size of 1024 each, resulting in 39 frames as explained in section 2.2. Each frame of 1024 samples are sub-divided into 16 sub-frames for processing, each sub-frame has 64 samples. Flow representation of the approach is shown in figure 8.

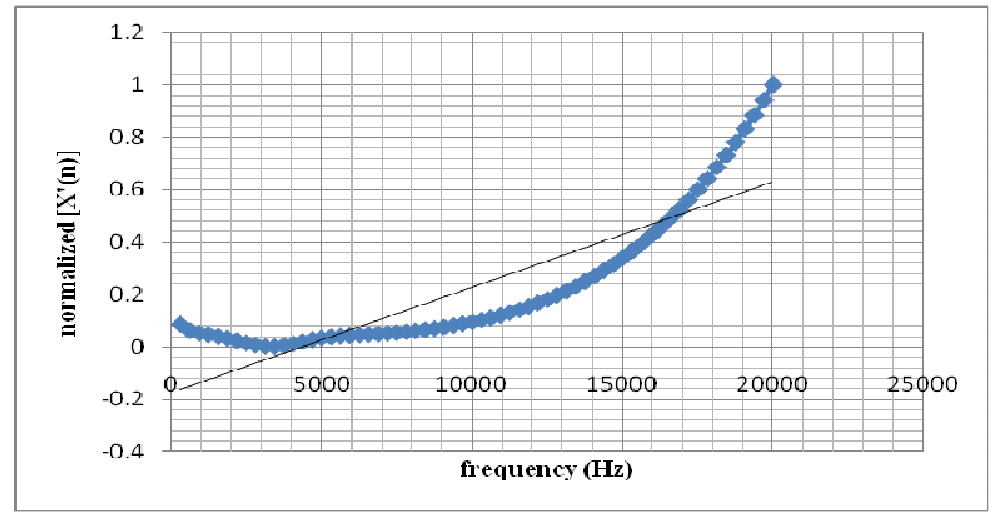

Fig. 6: Normalized vectors $\mathrm{V}_{\mathrm{s}}$ frequency $(\mathrm{Hz})$.

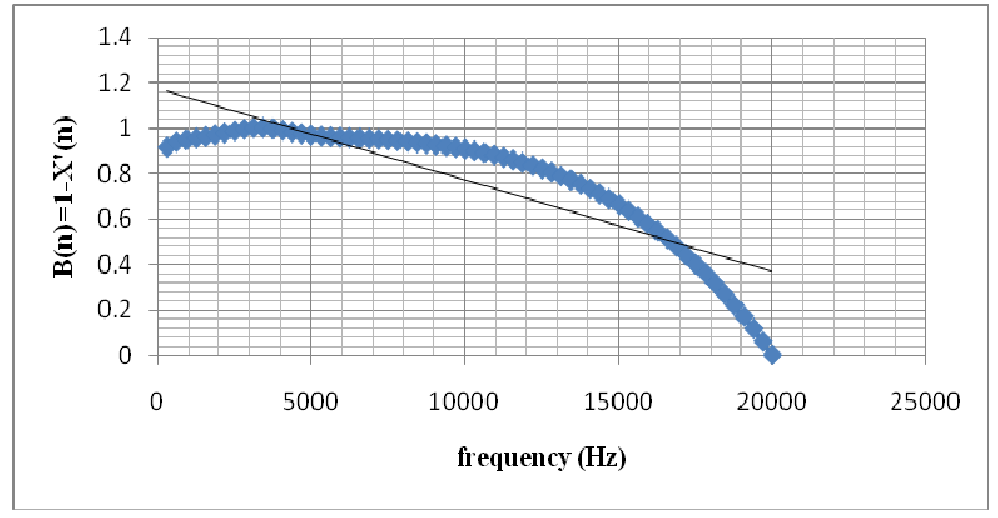

Fig. 7: Weighted Curve.

Obtain the magnitude of each sample by computing 64 point FFT of captured noise signal and compute the loudness level of varying background noise. The magnitude of frequency samples obtained by computing FFT is multiplied by 64 point weighed curve on sample basis and noise energy or noise loudness is calculated using the equation,

$$
\text { Loudness }=(1 / N) \sum_{i=0}^{N-1} 10\left(\log _{0}(R(i))^{2}\right)
$$

where

$$
R(i)=\sum_{n=0}^{N-1}(M(n) * B(n))
$$

$\mathrm{M}(\mathrm{n})=$ Magnitude of FFT samples

$\mathrm{B}(\mathrm{n})=1-\mathrm{X}^{\prime}(\mathrm{n})$, weighted $\mathrm{N}$ samples derived from figure 7 
Compute the magnitude of down-linked speech samples (with Fs $=8 \mathrm{kHz}$ ) using 64 point FFT for 16 sub-frames and calculate the energy (frame wise) of speech samples using equation 1. The energy of the speech and noise signals computed (figure 8) is compared and gain is derived for enhancing the degraded speech signal as explain in section 2. For deriving the gain equation 3 is used. Present frame gain is compared with previous frame gain if the difference is more, then current frame gain is adjusted to avoid sudden change in the output levels to avoid click \& pop noise which fatigue the ears. Updated gain is multiplied with speech (element by element) signal in time or frequency domain (multiplying by constant is a linear function) to get enhanced speech signal. If the enhanced/amplified value of speech signal exceeds the maximum loudness of the loud speaker then end capping can be performed depending on minimum and maximum values computed using equations 4 and 5 as explained in section 2.1.

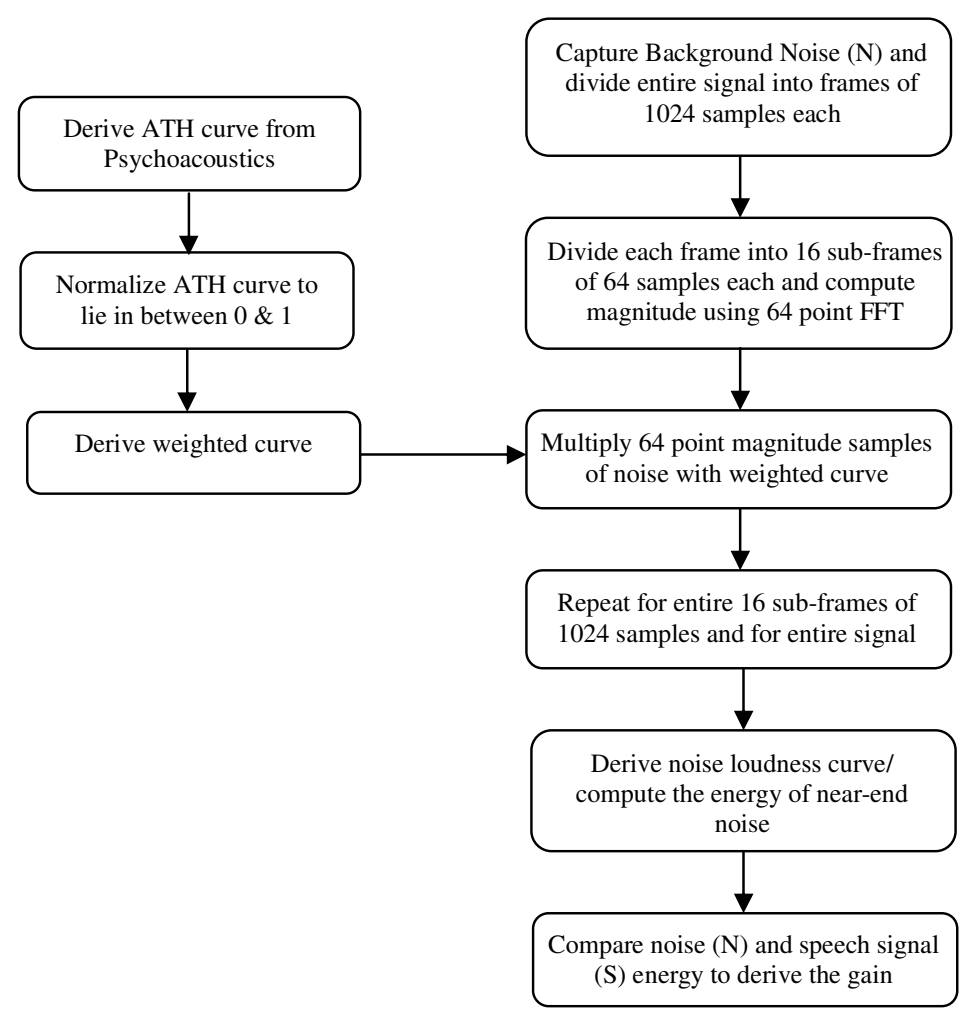

Fig. 8: Flow diagram of frequency domain approach using psychoacoustic.

\subsection{Experimental Results}

To illustrate a speech signal (Fig. 2(a)) and varying noise signal (Fig. 2(b)) of 16-bit PCM with sampling rate of $8 \mathrm{kHz}$ with duration of 5 seconds is captured using an audio editor tool, Gold Wave and saved in .wav format. The captured speech signal has 40000 samples; total samples are further divided into frame size of 1024 each, resulting in 39 frames. Figure 2(d) reflects the variation in the amplification of speech signal based on changing noise signals. Speech signal can be further enhanced by increasing the gain control, E in equation 3 as desired. 
International Journal of Wireless \& Mobile Networks (IJWMN) Vol. 5, No. 1, February 2013

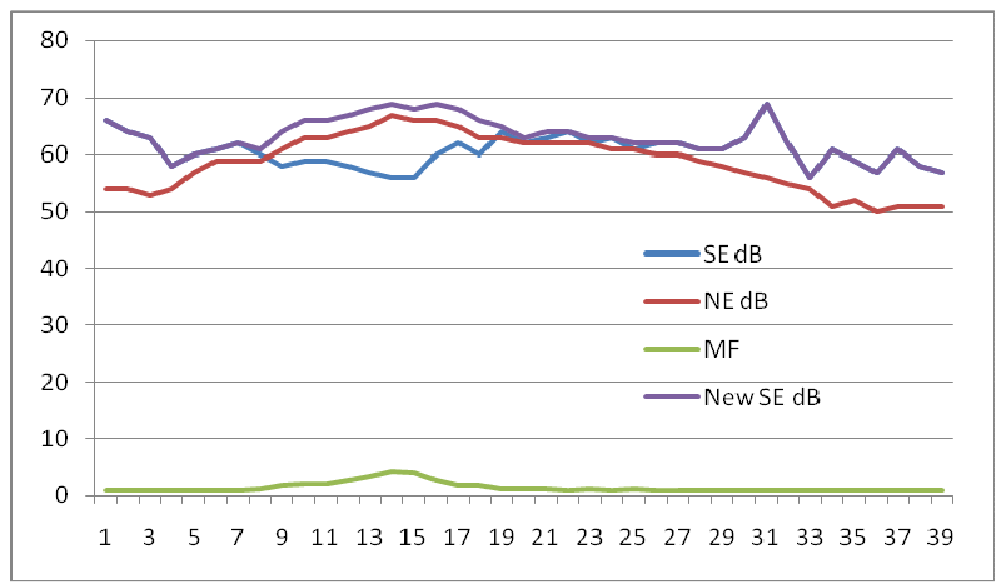

Fig. 9: Variation of speech, noise, enhanced speech signal and gain/MF using frequency domain approach.

The obtained results are plotted in figure 9. Number of frames is indicated in $\mathrm{x}$-axis and $\mathrm{y}$-axis indicates energy of the speech, noise $\&$ enhanced signal and variation of gain w.r.t. speech and noise signal. For a maximum gain of 4 the speech signal energy is enhanced by $13 \mathrm{~dB}$.

\section{Evaluation OF Both the Methods}

The gain derived from both the time and frequency domain (using psychoacoustic) approach is plotted in figure 10. Results indicate that later approach promises minimal gain required for speech enhancement, which is more realistic with reference to human audibility since psychoacoustic was involved. Complexity involved in the processing the samples will more in the latter approach because of frequency transformations (FFT) involved as compared to earlier approach. Algorithm enhances only the selected frequency components hence amplification required for enhancing speech signal is minimal which reduces the battery power as well as ears will not get fatigue.

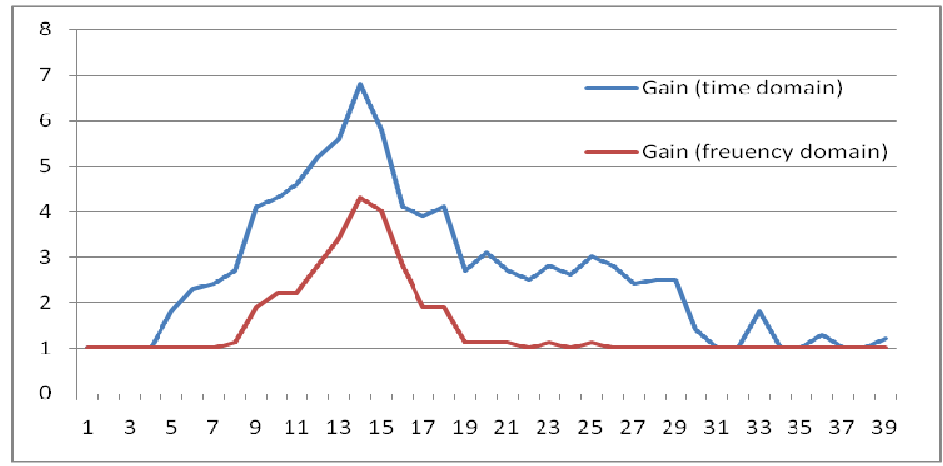

Fig. 10: Variation of gain/MF in time and frequency domain approach.

\section{CONClusions}

In this contribution we presented an efficient algorithm to enhance the speech signals in presence of noisy background noise. The paper discusses the implication of background noise on signal perception by the user and necessity of this problem needs to be solved for extended user experience and growth of mobile technology. The paper also discusses avenues to mitigate 
International Journal of Wireless \& Mobile Networks (IJWMN) Vol. 5, No. 1, February 2013

the effect of such noise by gain adjustment of speech signal according to noise variations. Experimental results are presented for a few combinations of parameters against the varying background noise, highlighting acceptable gain of the signal. Results indicate that gain obtained is adaptive and varies w.r.t. change in speech and noise signal. The proposed system is capable of tracking change in the user perception levels with time. The gain derived from frequency domain approach is more realistic because derived gain uses the masking properties of the human auditory system. Simulation results were verified using an audio editor tool Gold Wave v5.58, compiled with Eclipse $\mathrm{C} / \mathrm{C}++$ IDE and implemented on Beagle-Board-xM (OMAP3530) platform.

\section{ACKNOWLEDGEMENTS}

The authors would like to thank everyone, just everyone!

\section{REFERENCES}

[1] S. F. Boll, "Suppression of acoustic noise in speech using spectral subtraction", IEEE Transaction on Acoustic, Speech, Signal Processing, vol. ASSP -27, pp. 113-120, April. 1979.

[2] Y. Ephraim and D. Malah, "Speech enhancement using a minimum mean square error short-time spectral amplitude estimator", IEEE Transaction on Acoustic, Speech, Signal Processing, vol. ASSP-32, pp. 1109-1121, Dec. 1984.

[3] N Virag, "Single channel speech enhancement based on masking properties of human auditory system", IEEE Transaction on Speech and Audio Processing, vol. 7, pp. 126-137, 1999.

[4] Jeon Yu-young and Lee Sang-min, "A speech enhancement algorithm to reduce noise and compensate for partial masking effect", Journal of Central South University of Technology, vol. 18, issue 4, pp. 1121-1127, August 2011.

[5] Malihe hassani and M. R. Karami mollaei, "Speech enhancement based on spectral subtraction in wavelet domain", IEEE $7^{\text {th }}$ International Colloquium on Signal Processing and its Applications, pp. 366-370, March 2011.

[6] Bastian Sauert and Peter Vary, "Near End Listening Enhancement: Speech Intelligibility Improvement in Noisy Environments", in: Proceeding of International Conference on Acoustics, Speech, and Signal Processing (ICASSP), pp. 493-496, May 2006.

[7] Bastian Sauert, Gerald Enzner and Peter Vary, "Near End Listening Enhancement with Strict Loudspeaker Output Power Constraining", in: Proceeding of International Workshop on Acoustic Echo and Noise Control (IWAENC), pp. 01-04, Sept. 2006.

[8] Bastian Sauert and Peter Vary, "Near End Listening Enhancement Optimized with respect to Speech Intelligibility Index", in: Proceeding of European Signal Processing Conference (EUSIPCO) vol. 17, EURASIP. New York, Hindawi Publication, pp. 1844-1848, Aug. 2009.

[9] Bastian Sauert and Peter Vary, "Near End Listening Enhancement considering Thermal Limit of Mobile Phone Loudspeakers", in: Konferenez Elektronische Sprachsignal Verarbeitung (ESSV), Germany, vol. 61, pp. 333-340, 28-30 ${ }^{\text {th }}$ Sept. 2011.

[10]Jong Won Shin, Student Member, IEEE and Nam Soo Kim, Member, IEEE, "Perceptual Reinforcement of the Speech Signal Based on Partial Specific Loudness”, IEEE signal processing letters, vol. 14, No. 11, pp. 887 - 890, Nov. 2007.

[11]Jong Won Shin, Woohyung Lim, Junesig Sung and Nam Soo Kim, "Speech Reinforcement based on Partial Specific Loudness", in $8^{\text {th }}$ Annual Conference of International Speech Communication Association (ISCA), INTERSPEECH 2007, Antwerp, Belgium, 27-31 ${ }^{\text {st }}$ Aug. 2007. PP. 978-981. 
International Journal of Wireless \& Mobile Networks (IJWMN) Vol. 5, No. 1, February 2013

[12]Jong Won Shin, Yu Gwang Jin, Seung Seop Park and Nam Soo Kim, "Speech Reinforcement based on Partial Masking Effect", in: Proceeding of IEEE Transaction on Acoustic, Speech, Signal Processing (ICASSP), pp. 4401 - 4404, April 2009.

[13] Yi Hu and Philipos C. Loizou, "Incorporating a Psychoacoustic Model in Frequency Domain Speech Enhancement”, in IEEE signal processing letters, vol. 11 no. 2, February 2004.

[14] Eberhard Zwicker and Hugo Fastl, Psychoacoustics, Facts and Models. New York: Springer, 2007.

\section{Authors}

Premananda B. S. has completed his M.Tech. in Digital Electronics from V.T.U., Karnataka. He has published 12 papers in national, international conferences and journals. His areas of interests are in the field of Communication and VLSI.

Dr. Uma B. V. has obtained her M.E. in Digital Tech. \& Instrumentation and Ph.D. from V.T.U., Karnataka. She has published 20 papers in national, international conferences and journals. Her areas of interests are in the field of Communication and VLSI.
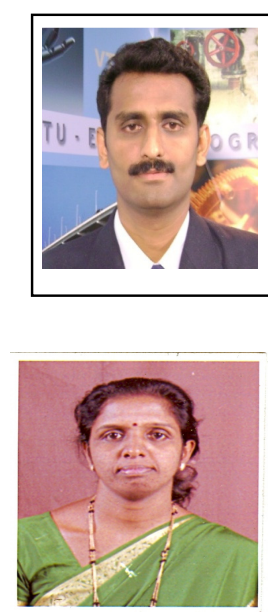